\title{
Recurrence of urinary tract infections in postmenopausal diabetic women using different antibiotics
}

\author{
Caroline Schneeberger ${ }^{1,2^{\star}}$, Ronald P. Stolk ${ }^{1}$, J. Hans De Vries ${ }^{3}$, Ron M. C. Herings ${ }^{4}$, \\ Suzanne E. Geerlings ${ }^{2}$ \\ ${ }^{1}$ Department of Epidemiology, University Medical Center Groningen, University of Groningen, Groningen, The Netherlands; \\ *Corresponding Author: carolineschneeberger@gmail.com \\ ${ }^{2}$ Department of Internal Medicine, Division of Infectious Diseases, Center for Infection and Immunity Amsterdam, Academic Medi- \\ cal Center (AMC), Amsterdam, The Netherlands \\ ${ }^{3}$ Department of Internal Medicine, Academic Medical Center (AMC), Amsterdam, The Netherlands \\ ${ }^{4}$ Utrecht PHARMO Institute, Utrecht, The Netherlands
}

Received 24 February 2012; revised 26 March 2012; accepted 12 April 2012

\section{ABSTRACT}

Objectives: Postmenopausal women with diabetes mellitus (DM) have an increased incidence of urinary tract infections (UTI) compared to women without DM. The aim of this study is to compare recurrence rates of UTI in postmenopausal women with DM after treatment with nitrofurantoin, the agent of first choice following the Dutch guidelines, with two other common prescribed antibiotics trimethoprim and norfloxacin. Methods: We used a PHARMO database with pharmacy dispensing data. A total of 8534 postmenopausal (>55 years) women with DM who received a first course of nitrofurantoin, trimethoprim or norfloxacin were included. The UTI recurrence rates after treatment with these three different antimicrobial agents were compared. Recurrence was defined as a second prescription for nitrofurantoin, trimethoprim or norfloxacin or a first with fosfomycin, amoxicillin, fluoroquinolones, or trimethoprim/sulfamethoxazole between 6 and 30 days after inclusion. Results: Postmenopausal women with DM had significantly more UTI recurrences when they were treated with nitrofurantoin (22.7\%) compared to trimethoprim (17.7\%) or norfloxacin (14.2\%) irrespective of the treatment duration. There was a trend that longer treatment duration was associated with higher recurrence rates. Conclusions: Postmenopausal women with DM had more UTI recurrences when they are treated with nitrofurantoin, agent of first choice, compared to trimethoprim or norfloxacin.
Keywords: Antibiotics; Urinary Tract Infections; Infectious Diseases; Treatment; Diabetes Mellitus

\section{INTRODUCTION}

Postmenopausal women with diabetes mellitus (DM) have an increased incidence and complication rate of urinary tract infections (UTI) compared to women without DM [1-3].

As far as we are aware, no randomized trials comparing different antibiotic treatments for UTI in women with DM are present. Because of the possible severity of UTI in patients with DM international guidelines recommend prolonged treatment with specific antimicrobial agents in these patients. However, most recommendations rely on expert's opinions [4].

The aim of this study is to compare recurrence rates of UTI in postmenopausal women with DM after treatment with nitrofurantoin, the agent of first choice following the Dutch guidelines with trimethoprim and norfloxacin which are also frequently used.

\section{METHODS}

We used a PHARMO database with pharmacy dispensing data. A total of 8534 postmenopausal ( $>55$ years) women with DM who received a first course of nitrofurantoin, trimethoprim or norfloxacin during the study period from the first of January 1999 until the first of January 2006 were included. All women with recurrent UTI because of known secondary or underlying abnormalities were excluded.

The UTI recurrence rates after treatment with these different antimicrobial agents were compared. Recurrence, the primary outcome, was defined as a second 
prescription for nitrofurantoin, trimethoprim or norfloxacin or a first with fosfomycin, amoxicillin, fluoroquinolones, or trimethoprim/sulfamethoxazole between 6 and 30 days after inclusion. Furthermore we compared recurrence rates between different treatment duration groups using $\chi^{2}$-test. The extended methods have been described elsewhere [5].

\section{RESULTS}

Postmenopausal women with DM had significantly more UTI recurrences when they were treated with nitrofurantoin (22.7\%) compared to trimethoprim (17.7\%) or norfloxacin $(14.2 \%)$ irrespective of the treatment duration. There was a trend that longer treatment duration with the same antimicrobial agent was associated with higher recurrence rates. For the majority of women nitrofurantoin or trimethoprim was prescribed 4 to 5 days and norfloxacin 6 to 7 days (Table 1 and Figure 1).

\section{DISCUSSION}

In this study we found that postmenopausal women

Table 1. Postmenopausal women with diabetes.

\begin{tabular}{ccc}
\hline Total Number $(N)$ & 8534 & $(67 ; 81)$ \\
Age $\left(\right.$ median $\left.\left(25^{\mathrm{e}} ; 75^{\mathrm{e}}\right)\right)$ & 75 & $(33.1 \%)$ \\
$N(\%)$ Use of insulin & 2826 & $(80.0 \%)$ \\
$N(\%)$ Use of oral antidiabetics & 6828 & \\
Patients using: & & $(43.8 \%)$ \\
$N(\%)$ nitrofurantoin & 3737 & $(41.0 \%)$ \\
$N(\%)$ trimethoprim & 3499 & $(15.2 \%)$ \\
$N(\%)$ norfloxacin & 1298 &
\end{tabular}

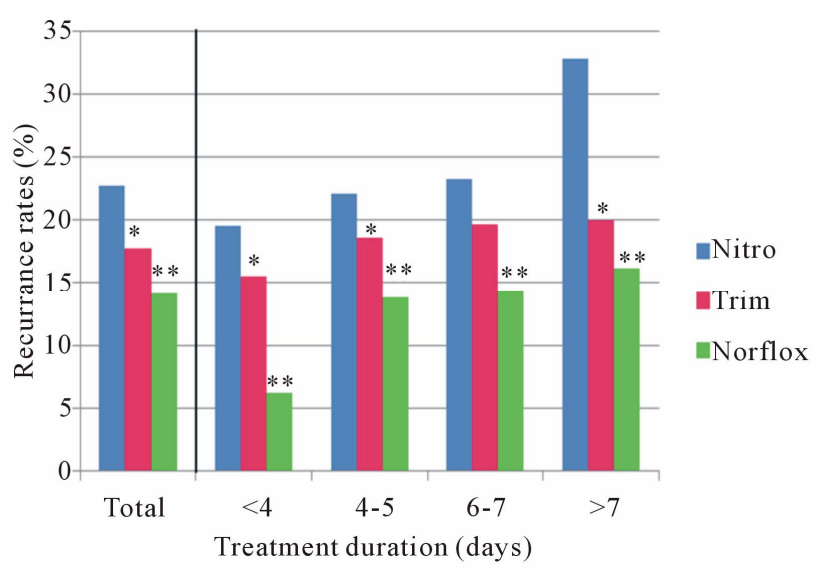

Figure 1. The recurrence rates by antibiotic type and by duration in postmenopausal women with diabetes. ${ }^{*}=P<0.05$ between recurrences rates nitro and trim. ${ }^{* *}=P<0.05$ between recurrences rates nitro and norflox. with DM had more UTI recurrences when they were treated with nitrofurantoin, the first choice agent, compared to trimethoprim or norfloxacin, irrespective of the treatment duration.

Our results differ from Lawrenson et al., who demonstrated no significant difference in recurrence rates between nitrofurantoin, trimethoprim or norfloxacin in premenopausal women aged 15 - 44 years with and without DM [6]. These differences can be explained by the study population of the present study that consisted of only postmenopausal women with DM. In addition it has been described before that trimethoprim and norfloxacin may reach therapeutic tissue concentrations in contrast to nitrofurantoin [7].

Treatment duration was not a primary outcome. Unexpectedly, we found that prolonged treatment with the same antimicrobial agent was associated with increasing recurrence rates. We assume that physicians prescribe a treatment with a longer duration for patients who clinically have a more severe UTI. Therefore, we cannot conclude that prolonged treatment is causally associated with higher recurrence rates.

These longer treatment durations were not in agreement with the recommendations in the national Dutch guideline 1999 in use during the study period. In this guideline it was recommended to treat lower UTI in women with and without diabetes in the same manner, namely with a 3-day oral antimicrobial regimen with either nitrofurantoin $100 \mathrm{mg}$ BID or trimethoprim once daily.

To our knowledge, this is one of the few studies that give us some insight in the treatment that is possibly required for UTI in postmenopausal women with DM, a special patient group which certainly needs more attention taking the increased incidence of UTI and associated complications in account [1-3].

The strength of this study is the large dataset with more than 8000 patients of an unselected population with a complete follow-up.

There are some limitations. Most important, clinical characteristics of the study population, like the severity of symptoms, were not available. Therefore, it was not possible to differentiate between complicated and uncomplicated, and upper and lower UTI. These could partly explain the lower recurrence rate in patients treated with norfloxacin, or the increased prolonged treatment. Combined with the observational design of the study causal conclusions cannot be made.

In conclusion, we showed that postmenopausal women with diabetes and a UTI had more UTI recurrences when they were treated with nitrofurantoin (agent of first choice) compared to trimethoprim or norfloxacin irespective of the treatment duration. We want to be modest to make conclusions, because cofounding by indication 
might have influenced these results. However, the results of this study emphasize that the optimal treatment of UTI in postmenopausal women with DM is not known. Randomized controlled trials with different treatment strategies are warranted to answer the question what the optimal treatment strategy is for UTI in postmenopausal women with DM.

\section{REFERENCES}

[1] Nicolle, L.E. (2005) Urinary tract infection in diabetes. Current Opinion in Infections Disease, 18, 49-53. doi:10.1097/00001432-200502000-00009

[2] Shah, B.R. and Hux, J.E. (2003) Quantifying the risk of infectious diseases for people with diabetes. Diabetes Care, 26, 510-513. doi:10.2337/diacare.26.2.510

[3] Carton, J.A., Maradona, J.A., Nuno, F.J., Fernandez-Alvarez, R., Perez-Gonzalez, F. and Asensi, V. (1992) Diabetes mellitus and bacteraemia: A comparative study be- tween diabetic and non-diabetic patients. European Journal of Medicine, 1, 281-287.

[4] Nicolle, L.E. (2001) A practical guide to antimicrobial management of complicated urinary tract infection. Drugs and Aging, 18, 243-254. doi:10.2165/00002512-200118040-00002

[5] Schneeberger, C., Stolk, R.P., Devries, J.H., Schneeberger, P.M., Herings, R.M. and Geerlings, S.E. (2008) Differences in the pattern of antibiotic prescription profile and recurrence rate for possible urinary tract infections in women with and without diabetes. Diabetes Care, 31, 1380-1385. doi:10.2337/dc07-2188

[6] Lawrenson, R.A. and Logie, J.W. (2001) Antibiotic failure in the treatment of urinary tract infections in young women. Journal of Antimicrobial Chemotherapy, 48, 895-890. doi:10.1093/jac/48.6.895

[7] Charalabopoulos, K., Karachalios, G., Baltogiannis, D., Charalabopoulos, A., Giannakopoulos, X. and Sofikitis, N. (2003) Penetration of antimicrobial agents into the prostate. Chemotherapy, 49, 269-279. doi:10.1159/000074526 